$3-2010$

\title{
Modeling Disk Cracks in Rotors by Utilizing Speed Dependent Eccentricity
}

Andrew L. Gyekenyesi

Ohio Aerospace Institute

Jerzy T. Sawicki

Cleveland State University, j.sawicki@csuohio.edu

Wayne C. Haase

Aerogage, Inc.

Follow this and additional works at: https://engagedscholarship.csuohio.edu/enme_facpub

Part of the Mechanical Engineering Commons, and the Structures and Materials Commons How does access to this work benefit you? Let us know!

\section{Publisher's Statement}

JMEPEG (2010) 19:207-212 @ASM International DOI: 10.1007/s11665-009-9500-0

$1059-9495 / \$ 19.00$

\section{Original Citation}

Gyekenyesi, A., Sawicki, J.T., and Haase W.C., Modeling Disk Cracks in Rotors by Utilizing Speed Dependent Eccentricity, Journal of Materials Engineering and Performance, Springer New York, Vol. 19, No. 2, pp. 207-212, 2010.

This Article is brought to you for free and open access by the Mechanical Engineering Department at EngagedScholarship@CSU. It has been accepted for inclusion in Mechanical Engineering Faculty Publications by an authorized administrator of EngagedScholarship@CSU. For more information, please contact library.es@csuohio.edu. 


\title{
Modeling Disk Cracks in Rotors by Utilizing Speed Dependent Eccentricity
}

\author{
Andrew L. Gyekenyesi, Jerzy T. Sawicki, and Wayne C. Haase
}

\author{
(Submitted March 25, 2009)
}

\begin{abstract}
This paper discusses the feasibility of vibration-based structural health monitoring for detecting disk cracks in rotor systems. The approach of interest assumes that a crack located on a rotating disk causes a minute change in the system's center of mass due to the centrifugal force induced opening of the crack. The center of mass shift is expected to reveal itself in the vibration vector (i.e., whirl response; plotted as amplitude and phase versus speed) gathered during a spin-up and/or spin-down test. Here, analysis is accomplished by modeling a Jeffcott rotor that is characterized by analytical, numerical, and experimental data. The model, which has speed dependent eccentricity, is employed in order to better understand the sensitivity of the approach. For the experimental set-up emulated here (i.e., a single disk located mid-span on a flexible shaft), it appears that a rather sizable flaw in the form of a throughthickness notch could be detected by monitoring the damage-induced shift in center of mass. Although, identifying actual disk cracks in complex "real world" environments, where noncritical crack lengths are small and excessive mechanical and/or electrical noise are present, would prove to be rather challenging. Further research is needed in this regard.
\end{abstract}

Keywords aerospace, failure analysis, machinery, nondestructive testing

\section{Introduction}

In order to improve safety and reliability in propulsion systems, there is much interest in assessing the feasibility of utilizing real-time vibration data for detecting cracks in turbine engine disks. The concept of the particular approach addressed here is based on the fact that the development of a disk crack results in a distorted strain field within the component. This, in turn, causes a small deformation in the disk's geometry as well as a minute change in the system's center of mass when compared to the undamaged case. The geometric change and the accompanying center of mass shift can be indirectly characterized by monitoring the amplitude and phase of the first harmonic (i.e., the $1 \times$ component) of the vibration data. Spin pit experiments, full scale engine tests, as well as some analytical studies have been documented regarding the above crack detection methodology (Ref 1-4), although, none of them presented a well-controlled situation with a known damage state. Confidence in the approach could improve with the presentation of controlled experimental and/or numerical

Andrew L. Gyekenyesi, Ohio Aerospace Institute, OAI/NASA GRC, 21000 Brookpark Rd, MS 6-1, Brook Park, OH 44135; Jerzy T. Sawicki, Cleveland State University, Cleveland, $\mathrm{OH}$; and Wayne C. Haase, Aerogage, Inc., Sterling, MA. Contact e-mails: Andrew.L.Gyekenyesi@grc.nasa.gov, j.sawicki@csuohio.edu, and whaase@summitsafetyinc.com. studies that utilize and calibrate to a known damage state as well as using relevant system parameters. This would allow for a better understanding of the crack dependent vibration response for an actual rotor.

For this study, the Jeffcott rotor is utilized as the basis for developing a crack dependent model that utilizes parameters defined by numerical and experimental data. Mainly, the formulation includes an eccentricity vector that is dependent on speed, which is a modification of the traditional modeling approaches that assume a fixed eccentricity. In the end, the vibration response obtained from the newly developed model provides a relevant view of the sensitivity of the method at least in regard to the particular NASA GRC experimental facility that is being emulated.

\section{Modeling Approach}

The model based on the Jeffcott rotor, employs a flexible rotor composed of a centrally located, unbalanced disk attached to a massless elastic shaft. In turn, the shaft is mounted symmetrically on rigid bearings (see Fig. 1). For utilization of the above described crack detection scheme, the disk unbalance is formulated to allow for a speed dependent eccentricity vector (i.e., the location of the center of mass in respect to the geometric center). For the case explored here, the speed dependence of the eccentricity vector is due to a circumferential disk crack located approximately at the midradius of the disk that is expected to open with increasing centrifugal force. The function that describes the changing eccentricity is defined based on results from finite element simulations of a notched disk. Further details of the disk, the numerical analysis, and the experimental system of interest are provided in the next section. 


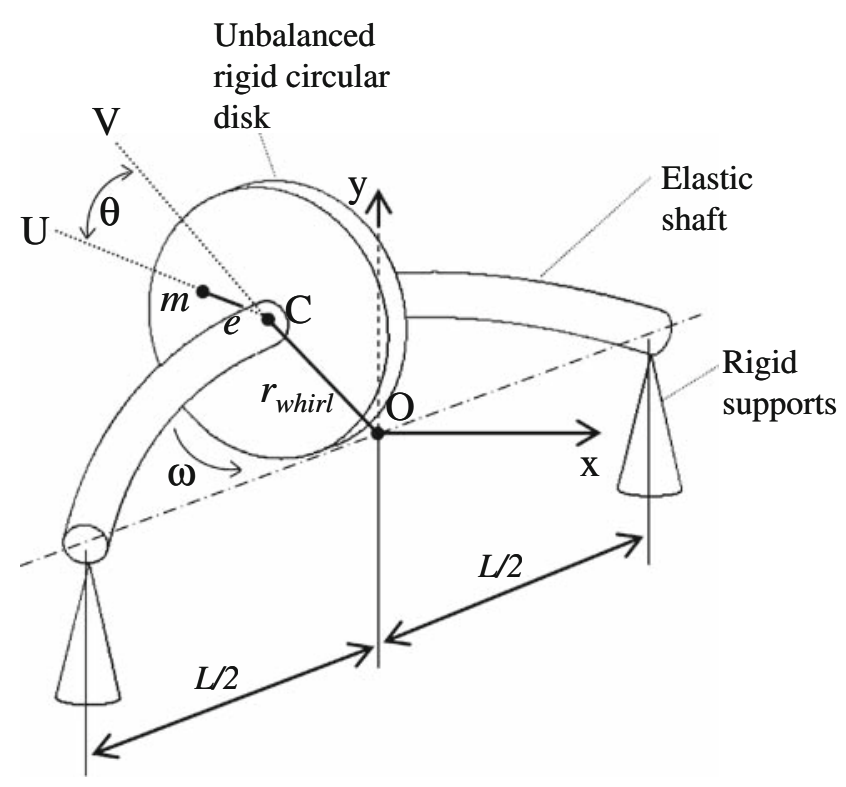

Fig. 1 Jeffcott rotor

The linear equations of motion in the $x$ and $y$ directions, running at constant speed, $\omega$, and having an eccentricity $e$, can be written as follows:

$\ddot{x}+2 \xi \omega_{\mathrm{n}} \dot{x}+\omega_{\mathrm{n}}^{2} x=e \omega^{2} \cos \omega t$

$\ddot{y}+2 \xi \omega_{\mathrm{n}} \dot{y}+\omega_{\mathrm{n}}^{2} y=e \omega^{2} \sin \omega t$

In Eq 1 and 2, $\xi$ represents the damping ratio, $c / c_{\text {critical }}$, and $\omega_{\mathrm{n}}$ is the system's natural frequency. Note that $c_{\text {critical }}$ is the amount of damping required to completely suppress free vibration; it is equal to $2 \sqrt{\mathrm{km}}$ and is assumed concentrated at the geometric center of the disk. The natural frequency, $\omega_{n}$, has a value equal to $\sqrt{k / m}$.

\subsection{Undamaged Case}

The steady-state solution for the undamaged case is as follows (Ref 5):

$x=X \cos (\omega t-\theta)$

$y=Y \sin (\omega t-\theta)$

where

$X=Y=\frac{e r^{2}}{\sqrt{\left(1-r^{2}\right)^{2}+(2 \xi r)^{2}}}$

and

$\theta=\tan ^{-1}\left(\frac{2 \xi r}{1-r^{2}}\right)$

Equations 3 and 4 represent the displacement of the geometric center of the disk, $O \vec{C}$, as seen in Fig. 1 . The phase angle, $\theta$, indicates that the rotor imbalance vector leads the whirl vector by the value defined in Eq 6 . Note that $r=\omega / \omega_{\mathrm{n}}$ is introduced for mathematical convenience. The solution shows that with large $r$, the amplitude of the whirl vector (defined by Eq 5) approaches $e$, while the phase angle approaches $180^{\circ}$.

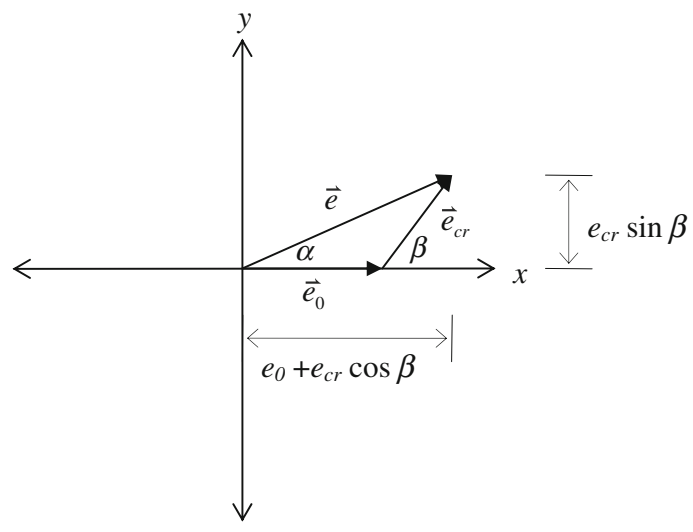

Fig. 2 The scenario of the eccentricity vector, $\vec{e}$, of a cracked rotor as the sum of an initial eccentricity vector, $\vec{e}_{0}$, and a crack-induced change vector, $\vec{e}_{\mathrm{cr}}$.

After surpassing the critical speed, the rotor aims to rotate about its center of mass.

\subsection{Speed Dependent Eccentricity}

Remembering that the eccentricity is a vector, any change in $\vec{e}$ is reflected in both the amplitude and angular response of the solutions for $x$ and $y$. Figure 2 shows the scenario of the eccentricity vector, $\vec{e}$, of a cracked rotor as the sum of an initial eccentricity vector, $\vec{e}_{0}$, and a crack-induced change vector, $\vec{e}_{\mathrm{cr}}$. The initial eccentricity, $\vec{e}_{0}$, is arbitrarily chosen along the $x$-axis while $\vec{e}_{\text {cr }}$ forms an angle $\beta$ with the $x$-axis. The amplitude of the total eccentricity vector, $\vec{e}$, and the angle in respect to the $x$-axis are as follows:

$|e|=\sqrt{e_{0}^{2}+e_{\mathrm{cr}}^{2}+2 e_{0} e_{\mathrm{cr}} \cos \beta}$

$\angle e=\alpha=\tan ^{-1} \frac{e_{\mathrm{cr}} \sin \beta}{e_{0}+e_{\mathrm{cr}} \cos \beta}$

If a crack-induced change in eccentricity is present, the $x$ and $y$ solutions take the form:

$x=\frac{r^{2} \sqrt{e_{0}^{2}+e_{\mathrm{cr}}^{2}+2 e_{0} e_{\mathrm{cr}} \cos \beta}}{\sqrt{\left(1-r^{2}\right)^{2}+(2 \xi r)^{2}}} \cos (\omega t-(\theta+\alpha))$

$y=\frac{r^{2} \sqrt{e_{0}^{2}+e_{\mathrm{cr}}^{2}+2 e_{0} e_{\mathrm{cr}} \cos \beta}}{\sqrt{\left(1-r^{2}\right)^{2}+(2 \xi r)^{2}}} \sin (\omega t-(\theta+\alpha))$

and

$(\theta+\alpha)=\tan ^{-1}\left(\frac{2 \xi r}{1-r^{2}}\right)+\tan ^{-1}\left(\frac{e_{\mathrm{cr}} \sin \beta}{e_{0}+e_{\mathrm{cr}} \cos \beta}\right)$

Note that the phase value defined by Eq 11 is now a sum of $\theta+\alpha$ as opposed to simply $\theta$ as in Eq 3, 4, and 6. The additional term, $\alpha$, modifies the direction of eccentricity due to the introduction of the crack vector $\vec{e}_{\text {cr. }}$. Also, realize that Eq 9 and 10 revert to Eq 3 and 4 when no crack-induced change vector is present. Next, utilizing finite element (FE) analysis, the variable amplitude of $\vec{e}_{\mathrm{cr}}$ is calculated as a function of rotational speed for the particular disk design and damage case that has been utilized in Ref 6 and 7 . 


\section{Numerical Analysis Defining Speed Dependent Eccentricity}

The FE analyses are described in detail in Ref 6. In summary, the goal was to understand the behavior of the isolated disk as a function of damage state and rotational speed. The damage state was in the form of a through-thickness, circumferential notch located at approximately mid-radius as shown in Fig. 3. The loading was due to centrifugal forces. Note that at the global level (i.e., vibration measurements), the notch satisfactorily emulates a crack. The disk was composed of a nickel alloy and had dimensions as follows; an outside diameter of $235 \mathrm{~mm}$ (9.25 in.); a bore thickness and outside rim thickness of 25.4 and $31.8 \mathrm{~mm}$ (1.00 and $1.25 \mathrm{in}$.), respectively; the thinnest portion of the web was $2.5 \mathrm{~mm}$ ( 0.10 in.); and the cross section and height dimensions of the blades were $31.8 \mathrm{~mm} \times 3.3 \mathrm{~mm} \quad(1.25$ in. $\times 0.13$ in.) and $8.4 \mathrm{~mm}(0.33 \mathrm{in}$.$) , respectively. During the design and damage$ study, the FE analyses of the disk were conducted using commercially available FE software. In the analyses, threedimensional brick elements were utilized with an auto-mesh feature to create the model. The numerical analyses were conducted for various notch lengths and disk speeds. Reference 7 describes the experimental results for the condition of a $30.5 \mathrm{~mm}$ (1.2 in.) circumferential length notch with $0.4 \mathrm{~mm}$ ( 0.015 in.) width subjected to a maximum rotation speed of 5400 revolutions per minute (rpm). This particular condition is the focus of this study.

Outputs from the finite element model (e.g., the mass matrix and node displacements while in the deformed state) were utilized to calculate the disk's shift in center of mass. Figure 4 shows the calculated shift in the center of mass as a function of rotational speed for the $30.5 \mathrm{~mm}$ (1.2 in.) notch. Based on the finite element simulations and a second-order polynomial curve fit, the amplitude for the speed dependent eccentricity concerning this particular notch condition is

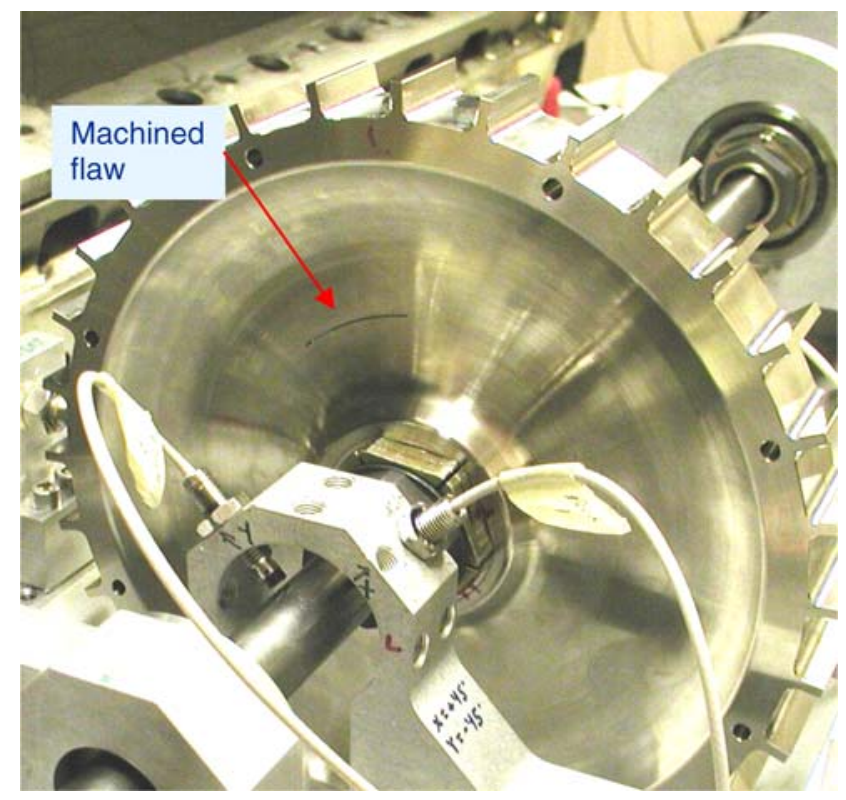

Fig. 3 Image of notched disk (30.5 mm [1.2 in.] notch) $e_{\mathrm{cr}}=c_{1} \omega^{2}+c_{2} \omega$

where $c_{1}$ and $c_{2}$ are $6.142 \times 10^{-11} \mathrm{~mm} / \mathrm{rpm}^{2}\left(2.418 \times 10^{-12}\right.$ in. $\left./ \mathrm{rpm}^{2}\right)$ and $1.162 \times 10^{-7} \mathrm{~mm} / \mathrm{rpm}\left(4.576 \times 10^{-9} \mathrm{in} . / \mathrm{rpm}\right)$, respectively. Next, the relationship obtained from the FEA, as represented in Eq 12, is utilized in Eq 9 and 10 in order to predict the response of a system containing a notch.

\section{Rotordynamic Response}

Table 1 summarizes the properties and calculated values utilized by the model. Values for bending stiffness, $k$, damping ratio, $\xi$, and initial eccentricity, $e_{0}$, were tailored in order to have the model match the experimental data concerning the undamaged disk in Ref 7. For instance, the bending stiffness of the shaft (bearings assumed rigid) was defined as $k=(86 E I) / L^{3}$ in order to obtain the proper natural frequency, while $\xi$ was chosen as 0.23 so as to have the appropriate whirl amplitude during critical speed (for small $\xi$, the whirl amplitude at critical speed is approximated as $\left.e_{0} / 2 \xi\right)$. The initial eccentricity was experimentally observed to be 0.001 in. (acquired from Bode plots: postcritical speed behavior, Ref 7). The upcoming Bode plots display the amplitude and phase of the whirl vector. The phase is based on Eq 11, while the whirl amplitude (i.e., the length of vector $O \vec{C}=\sqrt{x^{2}+y^{2}}$ as seen in Fig. 1b) is

$r_{\mathrm{whirl}}=\frac{r^{2} \sqrt{e_{0}^{2}+e_{\mathrm{cr}}^{2}+2 e_{0} e_{\mathrm{cr}} \cos \beta}}{\sqrt{\left(1-r^{2}\right)^{2}+(2 \xi r)^{2}}}$

Note that Eq 13 reveals an increasing amplitude due to the domination of the crack-induced shift in center of mass as speed induced centrifugal forces continue to open the crack/ notch.

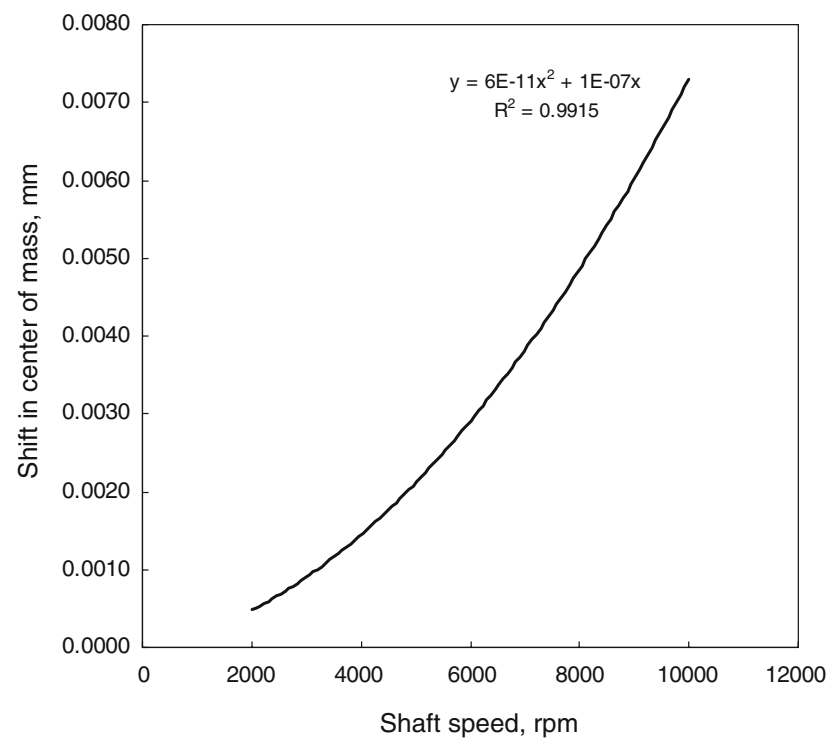

Fig. 4 Finite element analysis results defining amplitude change of the eccentricity vector due to speed dependent opening of a circumferential notch in the web area of the disk (30.5 mm [1.2 in.] notch). The second-order polynomial curve fit of the finite element-based results are indicated on the plot 


\begin{tabular}{lll}
\hline Property & \multicolumn{1}{c}{ Value } \\
\hline$E, \mathrm{~Pa}(\mathrm{psi})$ & $200 \times 10^{9}\left(29.0 \times 10^{6}\right)$ & Modulus of stainless steel shaft \\
$L, \mathrm{~m}$ (in.) & $0.749 \mathrm{~m}(29.5)$ & Length of shaft, bearing center to center \\
$R, \mathrm{~m}($ in.) & $0.0100(0.395)$ & Radius of shaft \\
$I, \mathrm{~m}\left(\mathrm{in}^{4}\right)$ & $7.9 \times 10^{-9}(0.0191)$ & Moment of inertia for shaft, $I=(1 / 4) \pi R^{4}$ \\
$k, \mathrm{~N} / \mathrm{m}(\mathrm{lb} / \mathrm{in})$. & $323 \times 10^{3}\left(1.86 \times 10^{3}\right)$ & Bending stiffness of shaft, $k=\left(86 E I / L^{3}\right.$ \\
$m, \mathrm{~kg}(\mathrm{lb} \mathrm{s} / \mathrm{in})$. & $4.876(0.0279)$ & Mass of nickel disk $($ shaft assumed massless $)$ \\
$\xi$ & 0.230 & Damping ratio, $c / c_{\text {critical }}$ \\
$\omega_{\mathrm{n}}, \mathrm{rpm}$ & 2466 & Natural frequency, $\omega_{\mathrm{n}}=\frac{60}{2 \pi} \sqrt{k / m}$ \\
$\omega_{\text {critical }}, \mathrm{rpm}$ & 2608 & Critical speed, $\omega_{\text {critical }}=\frac{60}{2 \pi} \sqrt{\frac{k}{m\left(1-2 \xi^{2}\right)}}$ \\
$e_{0}, \mathrm{~m}$ (in.) & $0.025 \times 10^{-3}(0.001)$ & Initial eccentricity \\
\hline
\end{tabular}

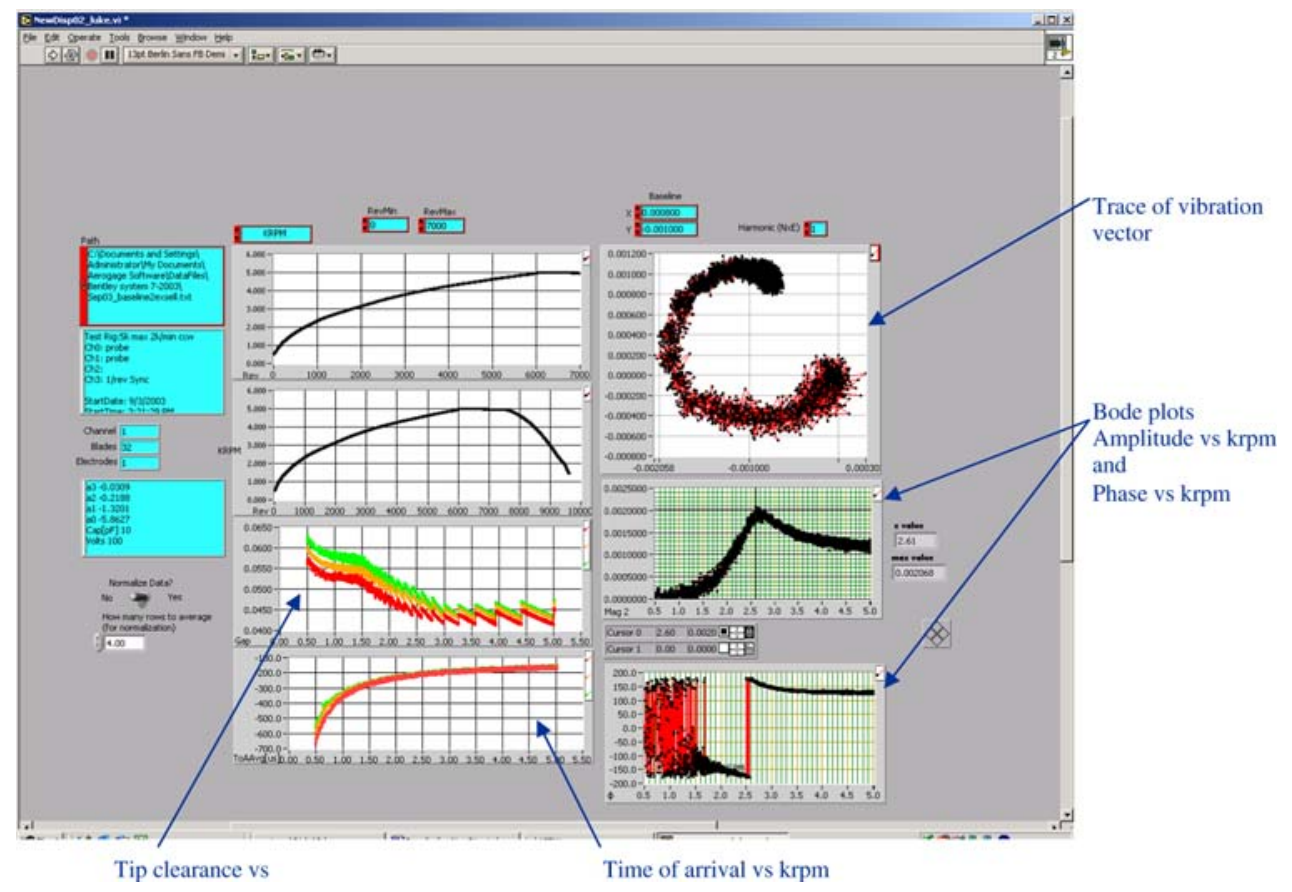

Fig. 5 Typical blade tip clearance data displayed using software associated with the capacitive sensors for the undamaged, baseline disk

Figure 5 displays typical experimental data for an undamaged disk (Ref 7). Because broadband, blade tip clearance measurements were used to produce the Bode plots, the data appear noisier than typical vibration measurements (e.g., shaft displacements using standard Eddy current probes and software). The experimentally derived critical speed and the associated maximum whirl amplitude are $2614 \mathrm{rpm}$ and $0.053 \mathrm{~mm}$ (0.0021 in.), respectively. Next, Fig. 6 shows the modeling results relating to various disk damage scenarios. The scenarios include the un-notched case (i.e., fixed eccentricity, $e$ ) as well as the case of a $30.5-\mathrm{mm}$ (1.2 in.) notch that causes the eccentricity to vary with speed. First, focusing on the un-notched case, the model deduced critical speed and maximum displacement are $2608 \mathrm{rpm}$ and $0.056 \mathrm{~mm}$ (0.0022 in.), respectively. Furthermore, when viewing both the experimental data and the modeling results for the un-notched case it is apparent that the whirl amplitude settles onto a value equal to the initial (i.e., fixed) eccentricity. Also, note the $180^{\circ}$ phase inversion that causes the system to rotate about its center of mass after the critical speed is traversed.

The other modeled scenarios include four orientations of the $30.5 \mathrm{~mm}$ (1.2 in.) notch in respect to the initial eccentricity vector (assumed parallel with the $x$-axis as seen in Fig. 2). Values for the angle $\beta$ include $0^{\circ}, 90^{\circ}, 180^{\circ}$, and $270^{\circ}$. As seen in Fig. 6, the largest deviations in amplitude, when compared to the un-notched case, occur for the $0^{\circ}$ and $180^{\circ}$ cases since the notch induced shift in center of mass is inline with the initial eccentricity. In Fig. 6, it is also apparent that at low speeds the notched rotor response is difficult to discern from the un-notched case since at lower speeds $e_{0}$ dominates and the influence of $e_{\mathrm{cr}}$ is minimal. As the critical speed is surpassed, the centrifugal forces and the associated crack/notch induced shift in center of mass start to dominate and the curves continue to separate. Furthermore, the final value of the phase inversion will be opposite of the crack vector value. For example, when a crack is located at $90^{\circ}$ from $e_{0}$ (note that $e_{0}$ is modeled at $0^{\circ}$ ), 

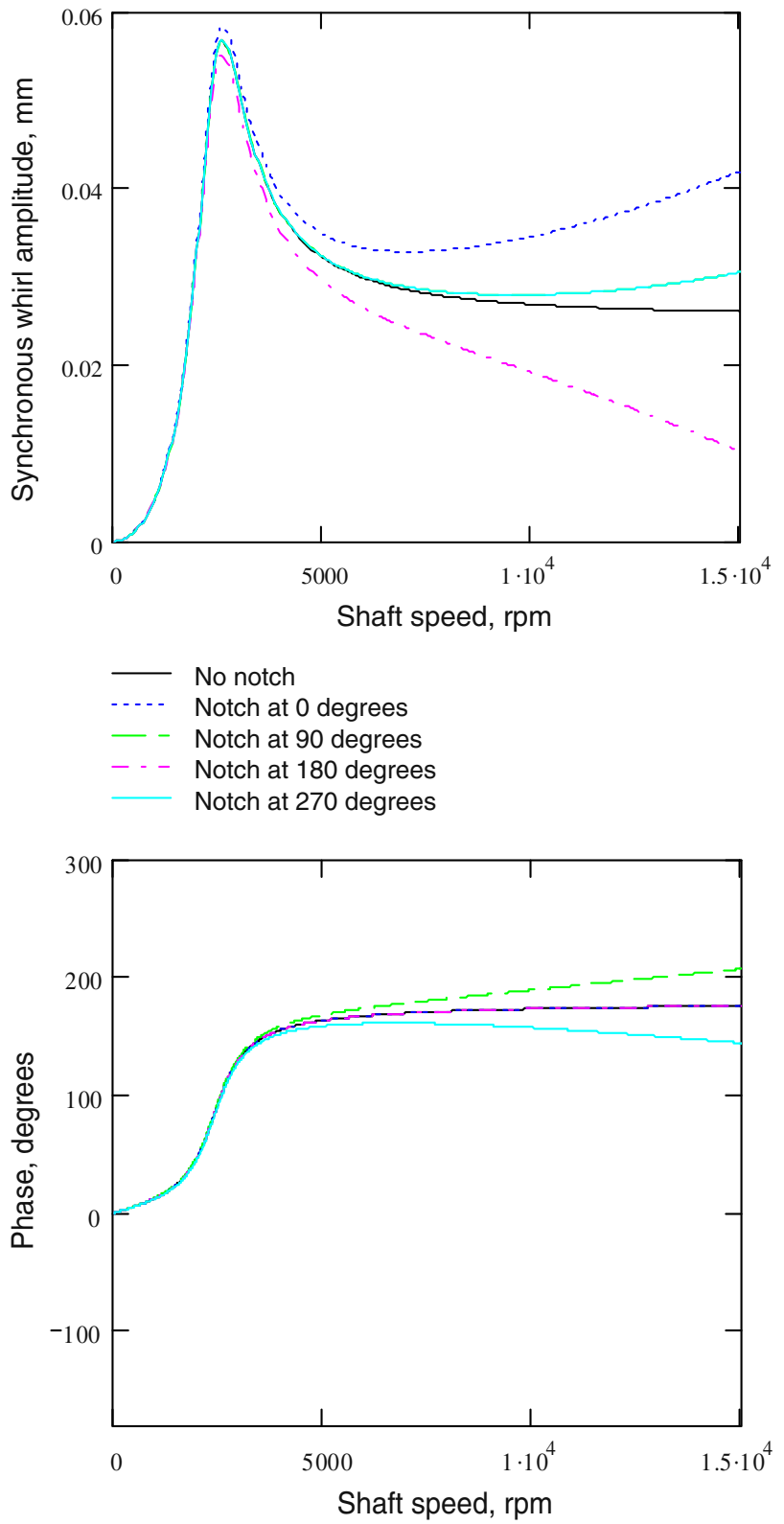

Fig. 6 Model generated Bode plots

the system desires to rotate about a value opposite of $90^{\circ}$, i.e., at $270^{\circ}$. Another interesting point involves the case of the crack at $180^{\circ}$. At about $20,000 \mathrm{rpm}$ (not shown), the amplitude equals $0^{\circ}$ and a $180^{\circ}$ phase inversion occurs as the amplitude starts to grow again in the opposite direction.

Figure 7 shows the amplitude and phase after subtracting the un-notched case from the four notched cases (using vector subtraction where the vector is defined by the amplitude and phase for the respective conditions). As expected, the vector change for all four subtractions collapses to a single amplitude plot. Note that mathematically the same subtraction result can be achieved by making $e_{0}=0$ in Eq 11 and 13 since the vector subtraction simply leaves the varying component (i.e., the notch induced eccentricity change, $e_{\mathrm{cr}}$ ). The phase difference plots show that the difference vectors, as explained above, should progress to be $180^{\circ}$ from the crack angle as the critical speed is surpassed.
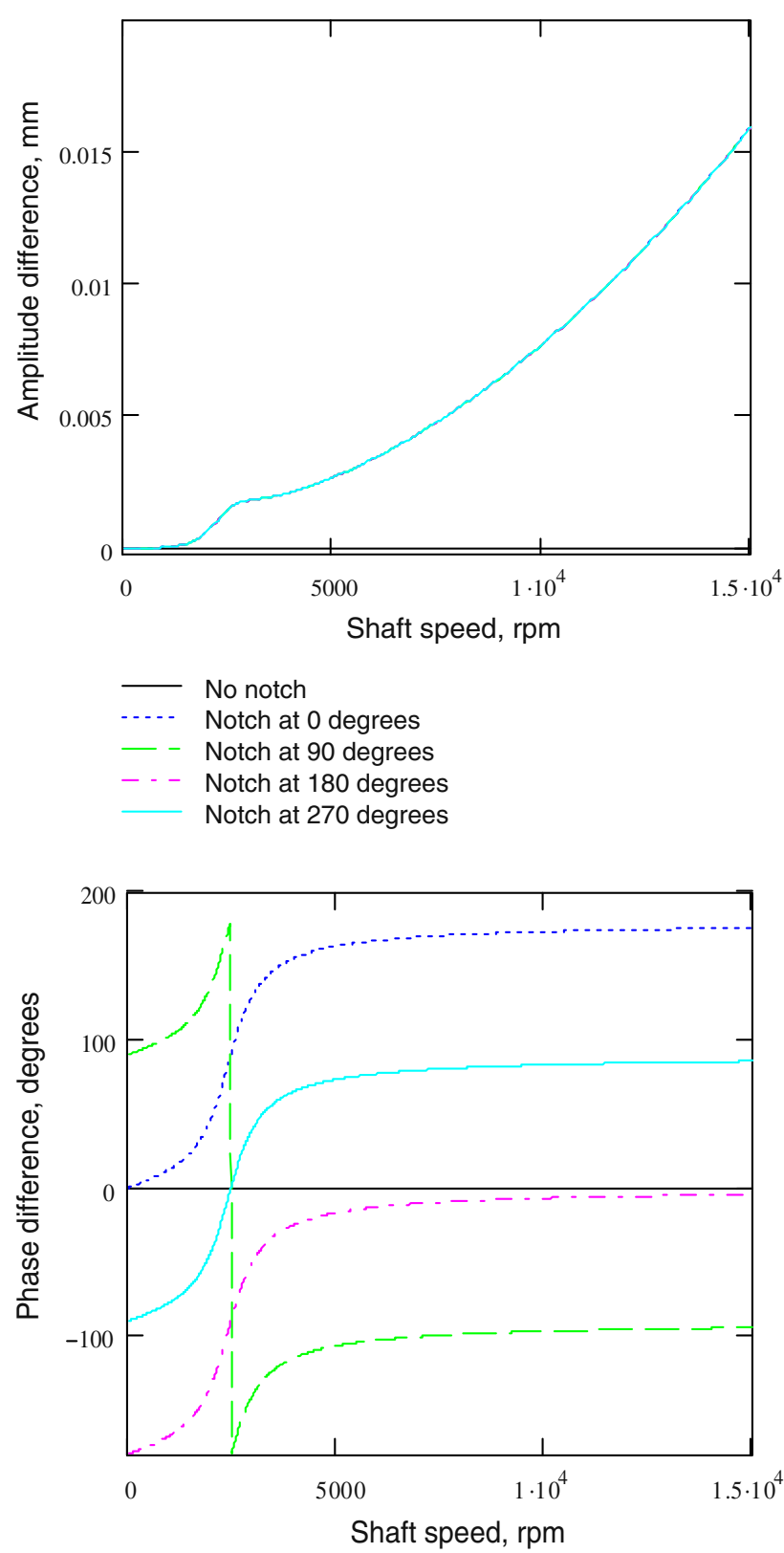

Fig. 7 Bode plots showing vector difference where the un-notch results are subtracted from each of the four notched conditions. For the amplitude graph, the data collapses to a single plot

Next, focusing on the specifics of the experimental system emulated by the model, it is seen that the amplitude change is predicted to be approximately $0.013 \mathrm{~mm}(0.0005 \mathrm{in}$.) at $12000 \mathrm{rpm}$ (see Fig. 7). This value is in the measurable regime of typical sensor systems for tip clearance or shaft vibrations. Note that the error bands observed in the experimental system of Ref 7 were $\pm 0.0025 \mathrm{~mm}$ $( \pm 0.0001$ in.). This was due to electrical and mechanical noise associated with the experimental hardware. Unfortunately, the maximum speed attained during the experiments was only $5400 \mathrm{rpm}$ (due to power issues with the drive motor). At such speeds, the notch induced shift in center of mass is only predicted to be $0.00254 \mathrm{~mm}$ (0.0001 in.) which is within the noise band. Hence, damage could not be discerned from the baseline data. 


\section{Conclusions and Future Directions}

This paper analyzed the feasibility of vibration-based structural health monitoring for detecting disk cracks in rotor systems. The analysis was accomplished by using a model with speed dependent eccentricity that was characterized by analytical, numerical and experimental data. Concerning the experimental set-up simulated here (a disk located mid-span on a flexible shaft), it appears that monitoring the crack-induced shift in the center of mass could be a viable approach for identifying disk cracks, although, the rotational speed needs to be far beyond the first critical. The approach can be utilized by observing before- and after differences in the Bode plots, or if baseline data are not available, then by monitoring the postcritical behavior for signs of crack-induced shifts with increasing speed. Functionally, the crack-induced shift depends on the square of the rotational speed. The approach lends itself well to this particular system since the first critical is rather isolated and the second critical occurs beyond $28,000 \mathrm{rpm}$ (Ref 6). Note that for the case studied, the whirl amplitude $(1 \times$ vibration component) deviation from the un-notched condition is approximately $0.013 \mathrm{~mm}(0.0005 \mathrm{in}$.) at $12,000 \mathrm{rpm}$ (first critical for the un-notched condition is $2608 \mathrm{rpm}$ ).

Identifying disk cracks in complex "real world" environments, where excessive mechanical and/or electrical noise are also present, would prove much more challenging. In such systems, multiple critical speeds may be closely spaced, thus placing the crack dependent response within the peaks and valleys of the critical speed behavior and making it very difficult to differentiate from the overall system performance. Modeling such systems and taking into account the variable eccentricity is a goal yet to be achieved. Further research as well as new approaches to signal processing are needed in this regard.

\section{Acknowledgments}

The authors would like to thank Dr. John Lekki and Dr. George Baaklini of the NASA Glenn Research Center at Lewis Field as well as the Integrated Vehicle Health Management Project within the Aviation Safety Program for providing the funds for this research effort.

\section{References}

1. W.C. Haase and M.J. Drumm, Detection, Discrimination, and Real-Time Tracking of Cracks in Rotating Disks, SPIE's NDE and Health Monitoring of Aerospace Materials and Civil Infrastructures, San Diego, California, March, 2002

2. H.E. Sonnichsen and B. Milatovic, "Detecting Anomalies in Rotating Components," United States Patent 6456945 B1, Sept 24, 2002

3. D.G. Lewicki, W.C. Emmerling, D. Altobelli, S. Seng, C. Frankenberger, and L. Fila, "TF41 Engine Fan Disk Seeded Fault Crack Propagation Test," NASA TM-2004-213092

4. H. Luo, H. Rodriguez, D. Hallman, and D. Corbly, "Disk Crack Detection for Seeded Fault Engine Test," NASA CR-2004-213069

5. J.M. Vance, Rotordynamics of Turbomachinery, Wiley-Interscience, New York, 1988

6. A.L. Gyekenyesi, J.T. Sawicki, and G.Y. Baaklini, "Vibration Based Crack Detection in a Rotating Disk: Part 1-An Analytical Study," NASA/TM-2003-212624

7. A.L. Gyekenyesi, J.T. Sawicki, and G.Y. Baaklini, "Vibration Based Crack Detection in a Rotating Disk: Part 2-Experimental Results," NASA/TM-2005-212624/PART2 